International Journal on Artificial Intelligence Tools

Vol. 0, No. 0 (2000) 000-000

(C) World Scientific Publishing Company

\title{
Episodes in Space: Qualitative Representation and Reasoning over Spatio-Temporal Objects
}

\author{
BAHER A. EL-GERESY, ALIA I. ABDELMOTY and CHRISTOPHER B. JONES \\ School of Computing, University of Glamorgan, \\ Treforest, Mid Glamorgan, Wales, CF37 1DL, U.K. \\ Email: bageresy,aiabdel,cbjones@glam.ac.uk
}

Received (received date)

Revised (revised date)

\begin{abstract}
There is growing interest in many application domains for the temporal treatment and manipulation of spatially referenced objects. Handling the time dimension in spatial databases can greatly enhance and extend their functionality and usability by offering means of understanding the spatial behaviour in time. Few works, to date, have been directed towards the development of formalisms for representation and reasoning in this domain. In this paper, a new approach is presented for the representation and reasoning over spatio-temporal relationships. The approach is simple and aims to satisfy the requirements of coherency, expressiveness and reasoning power. The topology of the domain is defined by decomposing spatio-temporal objects into representative components and relationships are defined between those components. Spatio-temporal reasoning is achieved by composing the relationships between the object components using constraint networks. New composition tables between simple spatio-temporal regions and between regions and volumes are also derived and used in the reasoning process.
\end{abstract}

Keywords: Spatial reasoning, spatio-temporal reasoning, spatio-temporal representation, spatial relations, qualitative reasoning, spatial databases.

\section{Introduction}

Time and space are primary dimensions in many application domains. Conceptually, time is an essential dimension for understanding and modeling space. For example, the distribution of land-based phenomena and activities is directly dependent on the transformation and diffusion processes that have led to the mutation and evolution of the geographic space ${ }^{1}$. Modeling both space and time covers a wide spectrum of applications, including, medical and physical sciences and geographic and multimedia information systems. For example, by modeling spatio-temporal objects and relations in a genetic database, we could pose queries of the sort, "What is the effect of suppressing gene $x$ on the growth of a group of cells in the first three weeks of embryonic development?".

Few works exist in the literature which address the problem of representing 
Episodes in Space...

spatio-temporal relationships, or reasoning over spatio-temporal domains. The complexity of the spatial dimension compounded with change over time hindered the progress in this domain. On the other hand, several approaches have been developed for the representation and reasoning with temporal relations ${ }^{2,3,4,5,6,7}$, and with spatial relations $8,9,10,11,12$. Treatment in space has proved to be more complex due to its multi-dimensionality, and the variety of types of spatial objects and spatial relations.

Recently, work has been developed for studying spatial change ${ }^{13}$ and spatiotemporal relations ${ }^{14,15,16}$. Integrating time and space in most of the approaches is based on approximating spatial objects by minimum bounding rectangles (mbr) and representing spatio-temporal objects by parallel-piped shapes (through extending the mbrs in time). The approach is limiting as only approximations of representations of spatio-temporal relations is possible. Other approaches exist ${ }^{17,18}$ which consider spatio-temporal objects by separate, start and end states, and ignore the behaviour of the object between those states. These approaches are valid only if the spatio-temporal objects co-exist in the same instance of time, and could fail otherwise.

In this paper, a new approach is developed for the representation and reasoning over spatio-temporal relationships. The approach is flexible and can be used with different levels of complexities in space and time. Objects are defined using a representative set of components in the spatio-temporal space. Relationships between objects are defined through relationships between their components. Spatio-temporal reasoning is treated essentially as a constraint network problem. Composition tables must be derived and used between objects of different types and dimensions. New spatio-temporal composition tables are derived using the method in ${ }^{19}$. In considering the temporal dimension, two new relationships were added to those table, i.e. an $n x n$ composition table is extended to an $(n+2)^{2}$ table. For simplicity, simple spatial regions are used to demonstrate the ideas in this paper, but the method is readily extendible to handle different types and complexities of spatial objects.

The rest of the paper is organised as follows. In section 2, some requirements are identified for the development of qualitative spatio-temporal formalisms. Section 3 defines the topology of the spatio-temporal domain by defining spatio-temporal objects and the concept of episodes and their different types. The representation of episodes and relationships in this domain are discussed in section 4 . In section 5 , a new approach is presented for spatio-temporal reasoning and examples are given to illustrate the complexity and effectiveness of the approach. Conclusions and a view on future directions is given in section 6 .

\section{Requirements}

Qualitative reasoning formalisms strives to satisfy two, sometimes conflicting, goals, namely, Expressiveness and Reasoning power. A tradeoff is usually sought between those goals. In the case of spatio-temporal reasoning, a third requirement 


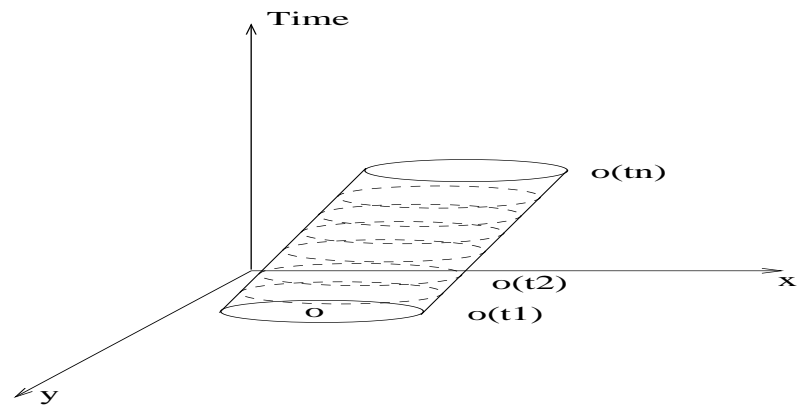

Figure 1: Dimensions of the Spatio-Temporal domain.

emerges, denoted Coherence. Coherence is the ability of the formalism to handle concepts from both the spatial and the temporal domains in a homogeneous and integrated fashion. The three requirements are described below:

1. Expressiveness: The proposed formalism should be able to handle different types and dimensions of spatio-temporal objects and different types and resolutions of spatio-temporal relations.

2. Reasoning power: A major goal in the development of the representation formalism is to form a basis for conducting spatio-temporal reasoning. Reasoning power is the ability of the formalism to derive non-explicit relationships between spatio-temporal objects given the knowledge of some existing ones, e.g, to deduce a relationship $R_{3}\left(O_{1}, O_{3}\right)$, given the relationships $R_{1}\left(O_{1}, O_{2}\right)$ and $\mathrm{R}_{2}\left(\mathrm{O}_{2}, \mathrm{O}_{3}\right)$.

3. Coherence: The formalism developed must unify the ordered one-dimensional temporal space and the unordered multi-dimensional spatial space. Hence, homogeneous concepts must be used to handle both domains for the development of a seamless formalism.

\section{The Problem Domain}

A spatio-temporal problem domain extends the usual 2D (or 3D) cartesian space by considering the time dimension. For simplicity, the presentation in this paper is confined to 2D spatial objects. However, the approach is applicable and extendible to three dimensional objects. The dimensions of our domain are represented in figure 1.

\section{A Spatio-Temporal Object}

In this paper spatial objects are considered to be functions of time. A spatial object $O_{i}$ is defined as,

$$
O_{i}=f(t)
$$




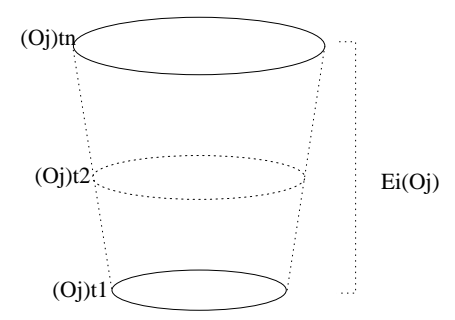

Figure 2: An episode defined as the combination of all object states in the time interval considered.

Examples of spatio-temporal objects in GIS include, boundaries of vegetation regions, administrative or political boundaries which change over time. The behaviour of objects with respect to time is not necessarily uniform or strict. Other approaches in this domain usually consider temporal behaviour of objects through their start and end states only and ignore any intermediate states. Those approaches assume implicit linear and uniform behaviour over time.

In this work, this constraint is not imposed. Instead, every consistent behaviour of a spatial object is considered separately within the duration of time when this behaviour can be described by a single function. This spatio-temporal behaviour shall be denoted, Episode of an object. For example, the spatial properties of an object may change linearly over a certain period of time, followed by an interval of no change, then followed by an interval of non-linear (e.g. cyclic*) change. This history of an object can, therefore, be described using three different episodes.

\section{An Episode}

An episode is used to describe the behaviour of a spatial object over a certain period of time.

A quantitative definition of an episode $e_{i}$ of an object $o_{j}$ over the time interval $t_{1}$ and $t_{2}$ is as follows.

$$
e_{i}\left(O_{j}\right)=\int_{t_{1}}^{t_{2}} f_{i}(t)
$$

$f_{i}(t)$ is a function that holds between $t_{1}$ and $t_{2}$. I.e. an episode is a representation of all the states of the object between times $t_{1}$ and $t_{2} . e_{i}\left(O_{j}\right)$ has a value $\left(O_{j}\right)_{t_{1}}$ at time $t_{1}$, which is the spatial extent of the object at time $t_{1}$, i.e. $\left(O_{j}\right)_{t_{1}}=[f(t)]_{t_{1}}$ as shown in figure 2 .

An episode is a coherent part of the history of an object. The full history of an object is a set of episodes.

$$
\text { history }\left(O_{j}\right)=\sum_{i=1}^{n} e_{i}
$$

*The change of coast-lines can be described by cyclic function while the phenomenon of continental drift can be described by a linear function. 

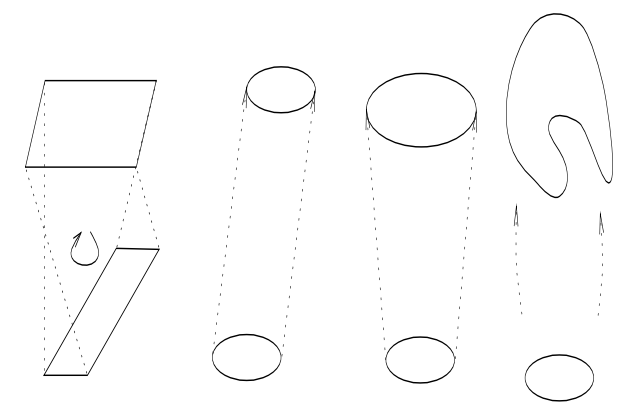

Figure 3: Some possible types of spatial change.

The function $f_{i}(t)$ can be used to qualitatively describe the different types of spatial changes that can an object may undergo.

\section{Types of Change}

Change can be classified according to the type and the rate of change. A spatial object can undergo any of four types of change in a spatio-temporal space, namely, translation (movement), rotation (change in direction), uniform or non-uniform scaling (change in size) or deformation (change in shape) or a combination of any of them. The different types of change are depicted in figure 3

The rate of change can be either 0 or $\neq 0$, corresponding to change or no change. A static episode is an episode through which the object does not undergo any change, i.e. its state remains constant. Hence, a static episode is defined as,

$$
\left[\frac{d\left(O_{j}\right)}{d t}\right]_{t_{1}}^{t_{2}}=0
$$

A dynamic episode is an episode through which the object has undergone one or more of the different types of change. It is defined as follows.

$$
\left[\frac{d\left(O_{j}\right)}{d t}\right]_{t_{1}}^{t_{2}} \neq 0
$$

Examples of types of spatio-temporal change are depicted in figure 4 in the case of scaling or change of size.

\section{Spatio-temporal Relations}

Spatio-temporal relations are studied between episodes of two objects and might be defined as functions of time.

$$
R_{k}\left(e_{i}\left(O_{j}\right), e_{m}\left(O_{k}\right)\right)=f(t)
$$

Two types of spatio-temporal relations can be identified:

Static Relations: A relation between two episodes is considered to be static if the relationship remains constant between the spatial objects involved during the 


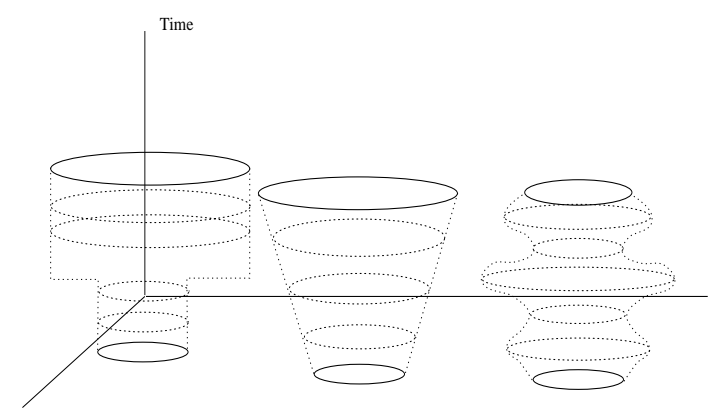

Figure 4: Some possible types of temporal change.

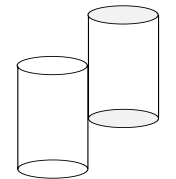

(a)

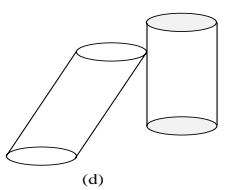

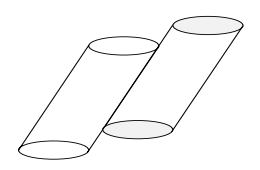

(b)

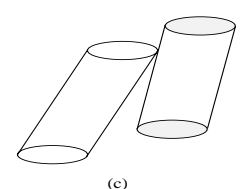

Figure 5: Examples of: (a) static episodes and static relations, (b) dynamic episodes and static relations, and (c) dynamic episodes and dynamic relations.

whole interval of time considered. For example, if $R_{1}\left(e_{i}, e_{m}\right)=$ touch at any instant in time between $t_{1}$ and $t_{2}$, then $R_{1}$ is considered to be static in this time interval. A static relationship can be defined as follows.

$$
\left[\frac{d R\left(e_{i}, e_{m}\right)}{d t}\right]_{t_{1}}^{t_{2}}=0
$$

Dynamic Relations: A spatio-temporal relation is considered to be dynamic if it is not static, i.e. the relation between the spatial objects changes during the interval of time considered. A dynamic relationship can be defined as follows.

$$
\left[\frac{d R\left(e_{i}, e_{m}\right)}{d t}\right]_{t_{1}}^{t_{2}} \neq 0
$$

Static relations may exist between either two static or two dynamic episodes. Dynamic relations exist only between two dynamic episodes or between a static and a dynamic episode. Examples of these relationships are shown in figure 5 .

\section{Representation of Episodes and Relationships between Episodes}




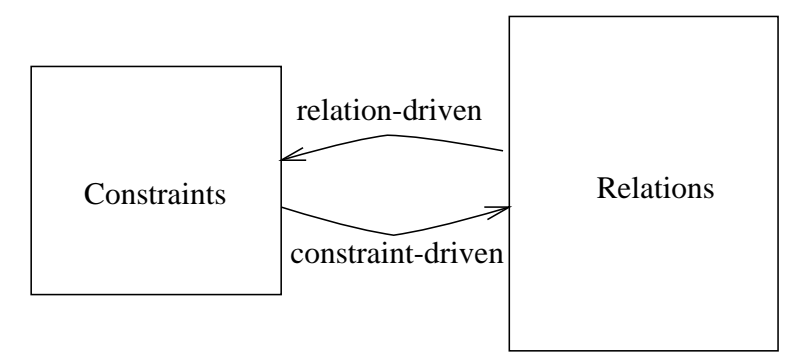

Figure 6: Different approaches to the representation of qualitative spatial relations.

A qualitative relation in space or time can be defined by the set of constraints that uniquely define it. Two approaches can be recognized for developing such formalisms, as shown in figure 6 , viz, a constraint-driven approach which starts by defining a general set of constraints based on which a set of relationships can be defined, and a relation-driven approach which starts by recognizing the set of relationships (intuitively), and then identifying the set of constraints necessary for their unique definition.

The work of Randell et al ${ }^{20}$ for defining topological relationships between convex regions is an example of the relation-driven approach. Another example is the interval algebra developed by Allen ${ }^{21}$. In the constraint-driven approach, the set of all possible constraints is first identified and different subsets of the constraints are combined combinatorially to define the different relations. Examples of this approach is the general formalism for defining topological relations in space and time ${ }^{19}$ using the intersection of object parts, and the definition of relations between intervals using the relations between their end-points developed by Vilain and Kautz 22 .

Although simple and intuitive in dealing with simple object, the relation-driven approach becomes very complex and error-prone when the domain of relations is large. Also, reasoning using this approach can easily become intractable as noted in ${ }^{23}$. The domain of spatio-temporal relations can be very large, making the task of adopting a relation-based approach almost impossible. Figure 7 illustrates a small subset of relations in this domain. A sound approach is needed to distinguish every relation in this figure.

A representation approach to the definition of spatio-temporal relations must satisfy the following requirements. It should be able to:

1. define the full set of temporal relations between episodes, namely, the set of 13 temporal relations between intervals and possible relations between intervals and points.

2. define the topological relations between the start states and end states of the two episodes considered. 


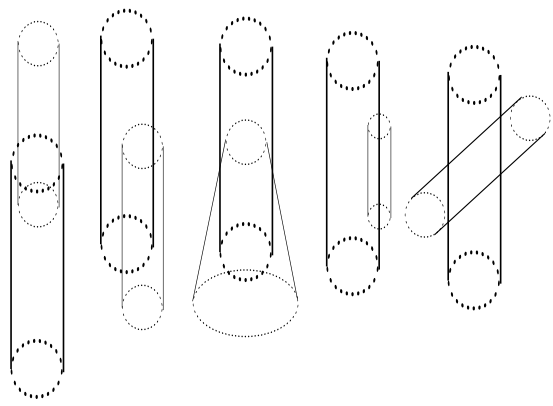

Figure 7: Some possible types of the overlap relationships between two volumes.

3. define the topological relations between the start and end states of one episode and the body of the other episode in the case of not-equal temporal relationship between episodes.

4. define the set of topological relations between the bodies of the two episodes under consideration.

Few works exist that address the problem of spatio-temporal representation. Other approaches deal only with the first three requirements above, and no works exist have yet addressed the fourth requirement. Chomicki and Revesz ${ }^{24}$ presented a qualitative geometric approach to the representation of spatio-temporal objects. Their method is based on qualitative description of objects as explicit geometries and the definition of translation as the only transformation function in time. The reasoning problem was not addressed, as object positions in space and behaviour in time were described quantitatively. Erwig et al ${ }^{6}$ proposed moving points and moving regions as the main types of spatio-temporal objects which they used as data types in the definition of spatio-temporal databases. Their work is mainly concerned with the identification and definition of spatio-temporal data types, as discrete (snap-shots) or continuous function, and didn't address the problems of representation of relations of reasoning in this domain.

A constraint-based approach is adopted in this paper where episodes are represented by a number of components that reflect their spatio-temporal characteristics. Relationships between episodes are then represented by the collective set of spatiotemporal relationships between their components. The constraint-based approach aims to avoid the need to classify and define a very large set of possible dynamic relationships.

\subsection{Representation of Episodes}

Episodes can be generally represented by three components as shown in figure 8, namely, two spatial regions, representing the extent of the episode at the start and the end of the interval occupied by the episode $\left(x_{s}\right.$ and $\left.x_{f}\right)$, and a three dimensional 


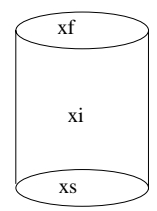

Figure 8: The three topological components of an episode: its start state $x_{s}$, its end state $x_{f}$ and its interior (intermediate states) $x_{i}$.

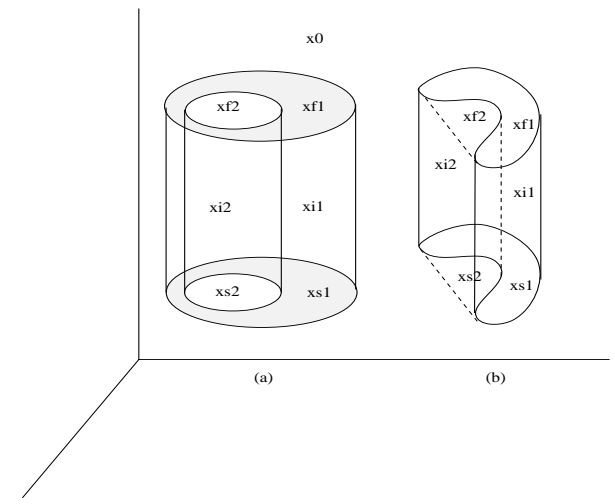

Figure 9: Examples of episodes for complex objects. (a) Region with a hole. (b) Concave region with a virtual cavity.

spatio-temporal volume $\left(x_{i}\right)$ representing the interior of the episode between $t_{s}$ and $t_{f}$. This representation is also applicable in the case of the $4 \mathrm{D}$ spatio-temporal episodes.

An episode type can be fully described using four components that correspond to the four types of possible changes that it may undergo, as described in the previous section. Hence, an episode can be described by a tuple $(L, R, C, D)$, where $L$ is the translation component, $R$, the rotation component, $C$, the scaling component and $D$, the deformation component. A value of 0 will be used to indicate no change and 1 to indicate change in that particular component. For example, $e_{1}(1,0,0,0)$ represents a dynamic episode that has undergone a translational change. The value of the tuple is representative of the nature of change, i.e., $e(L, R, C, D)=0$ represents no change or a static episode.

The method of representation used here captures both the spatial and temporal characteristics without imposing any restrictions on the spatial type of the episode or its temporal characteristics. For example, the same decomposition strategy can be used for representing more complex objects, such as, regions with holes, or regions with virtual components, as illustrated in figure 9 .

An episode is assumed to be embedded in an infinite, connected spatio-temporal space. It is assumed to be connected and no overlap is possible between its con- 


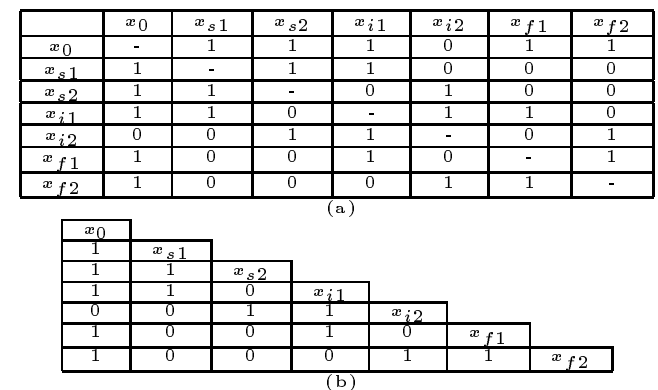

Figure 10: (a) Adjacency matrix of the shape in 9(a). (b) Half the symmetric adjacency matrix is sufficient to capture the representation of the episode.

stituting components. The topology of the episode and the embedding space can then be described by a matrix whose elements represent the connectivity relations between its components. This matrix shall be denoted adjacency matrix.

In figure 10(a), the representation of the episodes in figure 9 are represented. Each region is represented by two areal components $x_{s}$ and $x_{f}$ and infinite areal component $x_{0}$ representing the surrounding area. The fact that two components are connected is represented by a (1) in the adjacency matrix and by a (0) otherwise. Since connectivity is a symmetric relation, the resulting matrix will be symmetric around the diagonal. Hence, only half the matrix is sufficient for the representation of the object's topology and the matrix can be reduced to the structure in figure 10 (b). In the decomposition strategy, the complement of the object in question shall be considered to be infinite. The suffix $0\left(x_{0}\right)$ is used to represent this component.

\subsection{Representation of Spatio-Temporal Relations}

Spatio-temporal relations between episodes can be represented by the combined spatio-temporal relations between their constituting components. Distinction of topological relations is dependent on the strategy used in the decomposition of the objects and their related spaces.

For example, in figure 11(b), a simple relationship between two episodes of simple regions $x$ and $y$ is shown. This relationship is uniquely represented by coding the individual relationships between the different components in a matrix structure, denoted, the Component-Relations Matrix, as shown in 11(b).

It will now be shown how the representation method developed satisfy the requirements identified in section 4 above.

1. If the start or/and end components of two episodes are connected or intersect, i.e. not disjoint, then it can be inferred that they co-exist temporally. It is sufficient, in this case, to represent their spatial relationships. If the components are disjoint, then it is necessary to distinguish their temporal relationships.

Three possible types of disjoint relationships can be identified. These are 


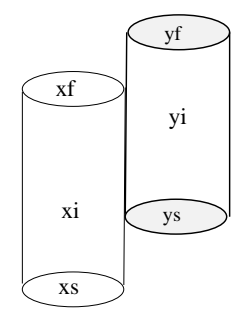

(a)

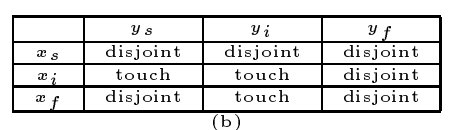

Figure 11: (a) A simple relationship between two episodes. (b) Its corresponding component-relations matrix.

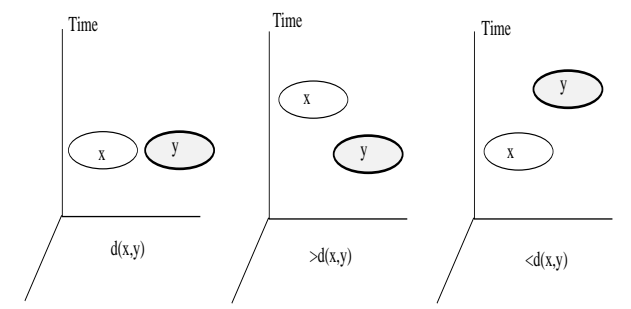

Figure 12: Different types of disjoint in the spatio-temporal domain.

illustrated in figure 12 .

(a) $d(x, y)$ : indicates that object $x$ is spatially disjoint from $y$ and both existed at the same time point.

(b) $<d(x, y)$ : indicating that object $x$ is spatially disjoint from $y$ and existed before $y$.

(c) $>d(x, y)$ : indicating that object $x$ is spatially disjoint from $y$ and existed after $y$.

Hence, the set of all possible temporal relationships between episodes, as well as the topological relations between start and end states (requirements 1 and 2) can be represented by the set of 10 spatio-temporal relations between states and end-states. The two temporal disjoint relationships will exist, irrespective of the complexity of the objects considered. Hence, in the case of simple convex regions, the set of eight topological relations is expanded to ten relationships and so on.

2. The third requirement studies the case of non-equal temporal relation between episodes. In this case there must exist a relation between the start or end state and the body of the other episode (between end states), i.e. a relationship between a $2 \mathrm{D}$ and a $3 \mathrm{D}$ object. This relation is equivalent to a $2 \mathrm{D}$ spatial relation between a $2 \mathrm{D}$ component of one episode (start and end 


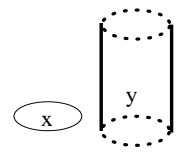

disjoint $\mathrm{d}(\mathrm{x}, \mathrm{y})$

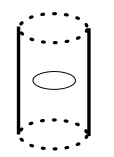

inside $\mathrm{i}(\mathrm{x}, \mathrm{y})$

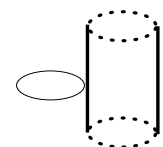

touch $\mathrm{t}(\mathrm{x}, \mathrm{y})$

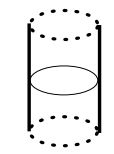

divide $\operatorname{dv}(\mathrm{x}, \mathrm{y})$
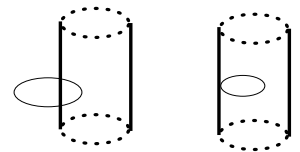

overlap o(x,y) coveredby $\mathrm{cb}(\mathrm{x}, \mathrm{y})$

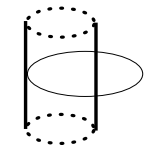

slice $\mathrm{s}(\mathrm{x}, \mathrm{y})$

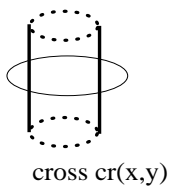

Figure 13: Different types of relationships between a region (representing an object state) and a volume (representing the interior of episodes).

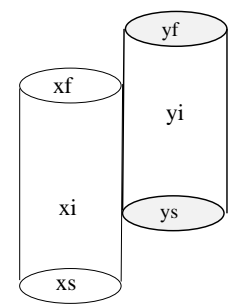

(a)

\begin{tabular}{|c|c|c|c|}
\hline & $y_{s}$ & $y_{i}$ & $y_{f}$ \\
\hline$x_{s}$ & $<d$ & $d$ & $<d$ \\
\hline$x_{i}$ & $t$ & $t$ & $d$ \\
\hline$x_{f}$ & $>d$ & $t$ & $<d$ \\
\hline
\end{tabular}

(b)

Figure 14: (a) A simple relationship between two episodes. (b) Its corresponding relation matrix, with different types of spatio-temporal disjointness.

states) and another 2D component of the other episode (cross-section of the body of an episode). If the objects considered are simple convex regions, then eight possible relationships can be distinguished. Different notations are used for naming the relations as shown in figure 13. Hence, the domain of relations in this case is the set of eight topological relations between convex regions.

3. In the fourth requirement, the topological relations between the bodies of the episodes, i.e. between two volumes, are considered. The full set of relationships between two convex volumes in space consist of eight topological relations similar to those in the case of two simple regions in 2D space. Hence, the six overlap relationships in figure 7 are uniquely distinguished using the method.

From the above, the relations matrix in figure 11 can be modified as shown in figure 14 .

Other examples of spatio-temporal relationships are shown in figure 15 along with their corresponding relations matrices.

Every instance of the component-relations matrix contains a collection of unique 


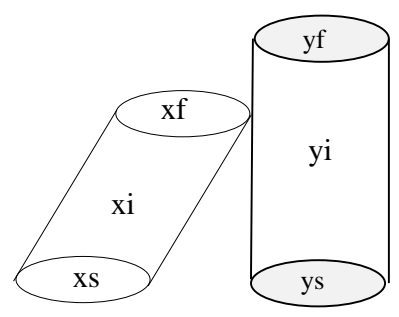

(a)

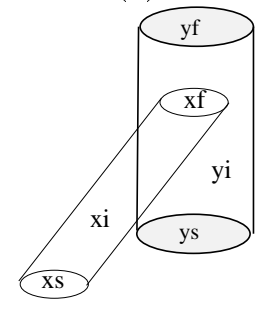

(c)

\begin{tabular}{|c|c|c|c|}
\hline & $y_{s}$ & $y_{i}$ & $y_{f}$ \\
\hline$x_{s}$ & $d$ & $d$ & $<d$ \\
\hline$x_{i}$ & $d$ & $d$ & $d$ \\
\hline$x_{f}$ & $>d$ & $t$ & $<d$ \\
\hline
\end{tabular}

(b)

\begin{tabular}{|c|c|c|c|}
\hline & $y_{s}$ & $y_{i}$ & $y_{f}$ \\
\hline$x_{s}$ & $<d$ & $d$ & $<d$ \\
\hline$x_{i}$ & $d$ & $o$ & $d$ \\
\hline$x_{f}$ & $>d$ & $c b$ & $<d$ \\
\hline
\end{tabular}

(d)

Figure 15: Examples of relations between episodes and their relations matrices.

relationships between the components, and hence, overall uniqueness and soundness of representation are guaranteed. I.e. every possible relationship between two episodes will have a corresponding matrix which uniquely distinguishes it.

\section{Spatio-Temporal Reasoning}

Spatio-temporal reasoning is carried out between episodes in a constraint-network fashion. The composition of relations is achieved by combining the Relation matrices, and propagating the relations between every pair of components of the two episodes.

The pre-requisites for carrying out the reasoning mechanism are as follows.

1. Composition tables for objects, or end-states. In our case, composition tables between simple regions need to be extended to include the different types of spatio-temporal disjointness. The extended table is shown in table 1.

2. Composition tables for end-states and bodies of the episodes, i.e. between regions and volumes. Four different tables are required to handle the different combination. The tables could be computed using the spatial reasoning approach developed in ${ }^{10}$. Note that all the entries in the composition table for region-volume and volume-region are disjunctive sets of all ten spatiotemporal relations between regions and hence the table can propagate no new information. The composition table between volume-region and region-volume 


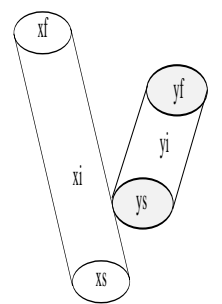

(a)

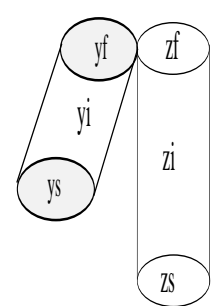

(b)

\begin{tabular}{|c|c|c|c|c|c|c|}
\hline$x_{f}$ & $x_{i}$ & $x_{s}$ & & $z_{s}$ & $z_{i}$ & $z_{f}$ \\
\hline$>d$ & $\mathrm{t}$ & $<d$ & $y_{s}$ & $>d$ & $\mathrm{~d}$ & $<d$ \\
\hline$d$ & $d$ & $d$ & $y_{i}$ & $d$ & $d$ & $d$ \\
\hline$>d$ & $d$ & $<d$ & $y_{f}$ & $>d$ & $d$ & $t$ \\
\hline
\end{tabular}

(c)

Figure 16: (a) Composing the relationships in (a) and (b). (c) Corresponding relation matrices.

is shown in table 2 as one example of the four tables.

3. Composition tables between the bodies of the episodes, i.e. between two volumes in our case. Since we can reduce the relations between two volumes to the same set of relations between two simple regions, the composition table in this case will be similar to that produced for simple regions.

Some other examples of the reasoning process follows.

\section{Example 1}

Consider the relationships between the episodes of objects $x, y$ and $z$ as shown in figure 16(a) and (b). Their Relation matrices are shown in 16(c).

Spatio-temporal reasoning is achieved using the following steps.

1. Propagate, systematically, the relationships between all pairs of components from the different episodes using the components of the common object.

Hence in the above example, the relationship between $x_{s}$ and $z_{s}$ is first derived using their relationships with $y_{s}, y_{i}$ and $y_{f}$. Then, relationships are derived between $x_{s}$ and $z_{i}$, followed by $x_{s}$ and $z_{f}$, and so on. As an example, the derivation of the relationship between $x_{s}$ and $z_{s}$ and $x_{i}$ and $z_{i}$ is given below.

(a) $R\left(x_{s}, z_{s}\right)$ :

From the matrices, the constraint network between the two components is shown in figure 17(a). Using the regions composition table, we have,

$$
\begin{aligned}
<d\left(x_{s}, y_{s}\right) \wedge>d\left(y_{s}, z_{s}\right) & \rightarrow \operatorname{All}\left(x_{s}, z_{s}\right) \\
d\left(x_{s}, y_{i}\right) \wedge>d\left(y_{i}, z_{s}\right) & \rightarrow \operatorname{All}\left(x_{s}, z_{s}\right) \\
<d\left(x_{s}, y_{f}\right) \wedge>d\left(y_{f}, z_{s}\right) & \rightarrow \operatorname{All}\left(x_{s}, z_{s}\right)
\end{aligned}
$$

By intersecting the resulting sets, we have the conclusion: $\operatorname{All}\left(x_{s}, z_{s}\right)$. 


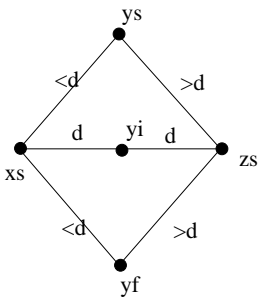

(a)

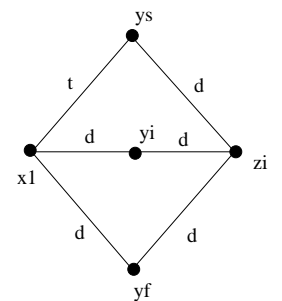

(b)

Figure 17: (a) (b)

(b) $R\left(x_{i}, z_{i}\right)$ :

From the matrices, the constraint network between the two components is show $\mathrm{n}$ in figure 17 (b). Using the regions composition table, we have,

$$
\begin{aligned}
t\left(x_{i}, y_{s}\right) \wedge d\left(y_{s}, z_{i}\right) & \rightarrow d\left(x_{i}, z_{i}\right) \vee t\left(x_{i}, z_{i}\right) \vee o\left(x_{i}, z_{i}\right) \vee c t\left(x_{i}, z_{i}\right) \vee c v\left(x_{i}, z_{i}\right) \\
d\left(x_{i}, y_{i}\right) \wedge d\left(y_{i}, z_{i}\right) & \rightarrow \operatorname{All}\left(x_{i}, z_{i}\right) \\
d\left(x_{i}, y_{f}\right) \wedge d\left(y_{f}, z_{i}\right) & \rightarrow \operatorname{All}\left(x_{i}, z_{i}\right)
\end{aligned}
$$

By intersecting the resulting sets, the composition yields a disjunctive set of relations: $d\left(x_{i}, z_{i}\right) \vee t\left(x_{i}, z_{i}\right) \vee o\left(x_{i}, z_{i}\right) \vee i\left(x_{i}, z_{i}\right) \vee c b\left(x_{i}, z_{i}\right)$. This result can be more concisely represented by the negation of the complement set of relations, i.e. $\neg e\left(x_{i}, z_{i}\right) \wedge \neg c t\left(x_{i}, z_{i}\right) \wedge \neg c v\left(x_{i}, z_{i}\right)$.

2. The full set compositions are refined and presented in a matrix, denoted, the composition matrix, as follows.

\begin{tabular}{|c|c|c|c|}
\hline & $z_{s}$ & $z_{i}$ & $z_{f}$ \\
\hline$x_{s}$ & All & All & $<d$ \\
\hline$x_{i}$ & All & $\neg i \wedge \neg c b \wedge \neg e q$ & $\neg c v \wedge \neg c t \wedge \neg e q$ \\
\hline$x_{f}$ & $>d$ & All & $>d$ \\
\hline
\end{tabular}

3. The constraints in the matrix are then combined and mapped to the resulting relation (or set of relations).

\section{Identifying possible sets of relations}

Soundness rules must be developed and used to map the composed relations matrix between the components of the episodes into a set of possible relations between the whole episodes. The development of such rules between objects of different types and dimensions is an ongoing area of research ${ }^{25}$ and is the subject of a different work.

For example, the composition of relations in the example above indicated that the possible relations between $x_{i}$ and $z_{i}$ may include $c t\left(x_{i}, z_{i}\right)$ or $c v\left(x_{i}, z_{i}\right)$. However, the resultant relations between $x_{i}$ and $z_{f}$ rules out those possibilities. 


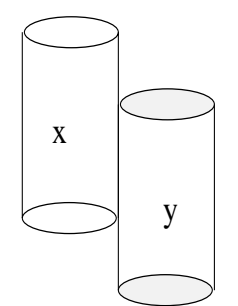

(a)

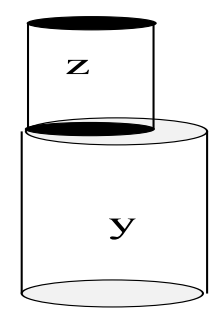

(b)

\begin{tabular}{|c|c|c|c|c|c|c|}
\hline$x_{f}$ & $x_{i}$ & $x_{s}$ & & $z_{s}$ & $z_{i}$ & $z_{f}$ \\
\hline$>d$ & $\mathrm{~d}$ & $>d$ & $y_{s}$ & $<d$ & $\mathrm{~d}$ & $<d$ \\
\hline$d$ & $t$ & $t$ & $y_{i}$ & $d$ & $d$ & $d$ \\
\hline$>d$ & $t$ & $<d$ & $y_{f}$ & $c v$ & $d$ & $<d$ \\
\hline
\end{tabular}

(c)

Figure 18: (a) Composing the relationships in (a) and (b). (c) Corresponding relation matrices.

The case of static episodes is special, since the spatial relations between them are preserved with respect to time.

\subsection{Spatio-temporal reasoning with Static Episodes and Relations}

A static episode is an episode where spatial extents of the object remains constant in time. A static relation between episodes implies the episodes being involved in a static spatial relation during their coexistence. The episodes in figure 5(a) are static and are involved in a static relationship. In this case, a rule that govern the inter-relationships between the episode components can be stated as follow. If the interior of one episode $e(i)_{1}$ has a relationship $R$ with the start $e(s)_{2}$ or end $e(f)_{2}$ of another episode $e_{2}$, then it must have the same relationship with the interior of that episode $e(i)_{2}$. I.e. $R\left(e(i)_{1}, e(s)_{2}\right) \vee R\left(e(i)_{1}, e(f)_{2}\right) \rightarrow R\left(e(i)_{1}, e(i)_{2}\right)$.

This rule can be incorporated in the reasoning process by deducing the relationship between $x_{i}$ and $z_{i}$ through the intersection of the set of relations between them and all the components of the other episode. I.e. $R\left(x_{i}, z_{i}\right)=R\left(x_{i}, z_{s}\right) \cap R\left(x_{i}, z_{i}\right) \cap$ $R\left(x_{i}, z_{f}\right) \cap R\left(z_{i}, x_{s}\right) \cap R\left(z_{i}, x_{f}\right)$.

\section{Example 2}

Consider the relationships between the episodes of objects $x, y$ and $z$ as shown in figure 18(a) and (b). Their Relation matrices are shown in 18(c).

Spatio-temporal reasoning is achieved by propagating, systematically, the relationships between all pairs of components from the different episodes using the components of the common object.

The full set compositions are refined and presented in the composition result matrix, as follows. 


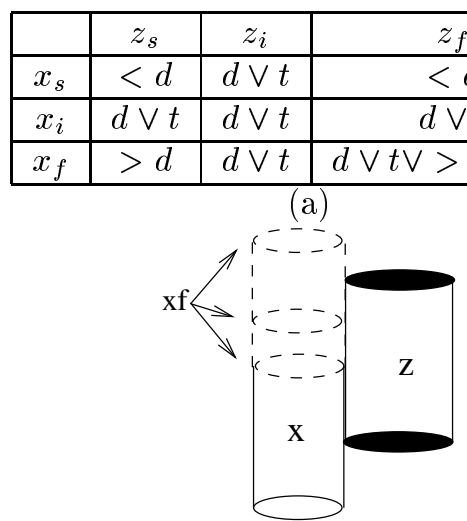

(b)

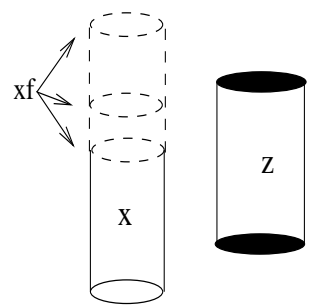

(c)

Figure 19: (a) The resulting matrix from the composition of relations in figure example 2 . (b) The possible types of resulting relations.

\begin{tabular}{|c|c|c|c|}
\hline & $z_{s}$ & $z_{i}$ & $z_{f}$ \\
\hline$x_{s}$ & $<d$ & $d \vee t \vee o \vee c t \vee c v$ & $<d$ \\
\hline$x_{i}$ & $d \vee t$ & $d \vee t \vee o \vee c t \vee c v$ & $d \vee t \vee o \vee c t \vee c v$ \\
\hline$x_{f}$ & $>d$ & All & All \\
\hline
\end{tabular}

The relationship between $x_{i}$ and $z_{i}$ is calculated as follows.

$R\left(x_{i}, z_{i}\right)=\{d \vee t \vee o \vee c t \vee c v\} \cap\{d \vee t \vee o \vee c t \vee c v\} \cap\{A l l\} \cap\{t \vee d\} \cap\{d \vee t \vee o \vee c v \vee c t\}=$ $\{d \vee t\}$.

It is noted that the matrix in not optimal, in the sense that some combinations may be impossible. Soundness rules need to be devised to exclude those cases. For static relations, it can be proved that if the initial propagation result is indefinite for the relations between the interiors of episodes and other elements, including bounding states, uncertainty must stem from the same disjunctive set of relations (with the addition of temporal disjointness). Hence, the same approach used for refining the relationship between $x_{i}$ and $z_{i}$ can be used other indefinite relations. Hence, by intersecting $R\left(x_{i}, z_{i}\right)$ with other indefinite results the composition result matrix can be restated as shown in figure 19(a). The matrix can correspond to one of six possible relationships shown in 19(b) and (c).

\section{Conclusions}

An approach was presented for the representation and reasoning over spatio-temporal relationships. A spatio-temporal object is defined and then used to define the notion of an Episode which is a collection of object states in a specific temporal interval defined between a start and an end state. The topology of the episode is defined by decomposing it into components representing its start, interior and end. Spatio-temporal reasoning is carried out over episodes by composing the relationships between their comprising components in a constraint network fashion. The 
following issues are also presented.

1. Different types of episodes are identified, namely, static and dynamic, reflecting the nature of change of spatio-temporal objects over time.

2. Spatio-temporal relationships are classified into static and dynamic according to whether spatial relationships between objects remain unchanged in time.

3. Spatial composition tables are extended by adding three different types of temporal disjointness, namely, $\langle d,>d$, and $d$. When objects are not disjoint, they are considered to be co-existing in time.

4. Different composition tables for relationships between bounding states and interiors of episodes are derived using the method in ${ }^{10}$.

5. In the case of static episodes, rules are defined and used to prune the compositions result into physically possible relationships.

The work in this paper is done within a framework of a research project which aims at the development of intelligent spatio-temporal databases ${ }^{10,19}$. Future work is directed towards three main issues.

- Defining complex dynamic episodes where more than one type of change occur, which is an important issue in simulation problem and other applications domains.

- Defining complex dynamic relations reflecting possible complex interaction between object.

- Utilising quantitative constraints, such as geometric configuration, in a hybrid quantitative-qualitative approach. This is important in applications domains, e.g. design and manufacturing, where topological constraints are not sufficient to define episodes and their inter-relationships.

\section{References}

[1] C. Claramunt and M. Theriault. Managing time in gis: An event-oriented approach. In Recent Advances on Temporal Databases. Springer Verlag, 1995.

[2] J.F. Allen. Maintaining Knowledge about Temporal Intervals. Artificial Intelligence and Language Processing, Communications of the ACM, 26:832-843, 1983.

[3] G. Edwards, P. Gagnon, and Y. Bedards. Spatio-Temporal Topology and Causal Mechanisms in Time Integrated GIS: From Conceptual Model to Implementation Strategies. In Proc. of the Canadian Conf. on GIS, pages 842-857, 1993.

[4] P. Terenziani and P. Torasso. Towards an Integration of Time and Causation in a Hybrid Knowledge Representation Fromalism. International Journal of Intelligent Systems, 9:303-338, 1994.

[5] M.F. Worboys. A Unified Model for Spatial and Temporal Information. The Computer Journal, 37(1):26-34, 1994. 
[6] M. Erwig, M. Schneider, and R.H. Guting. Temporal adn Spatio-Temporal Data Models and Their Expressive Power. Technical Report Informatik-Report 225, FernUniversitat, Hagen, 1997.

[7] A. Galton. A Critical Examination of Allen's Theory of Action and Time. Artificial Intelligence, 42:159-188, 1990.

[8] A.I. Abdelmoty and B.A. El-Geresy. A General Method for Spatial Reasoning in Spatial Databases. In Proceedings of the Fourth International Conference on Information and Knowledge Management (CIKM'95), pages 312-317. ACM press, 1995.

[9] A.I. Abdelmoty and M.H. Williams. Approaches to the Representation of Qualitative Spatial Relationships for Geographic Databases. In Advanced Geographic Data Modelling (AGDM'94), number 40 in Publications on Geodesy, pages 204-216. Netherlands Geodetic Commission, 1994.

[10] B.A. El-Geresy and A.I. Abdelmoty. Order in Space: A General Formalism for Spatial Reasoning. In Proc. of the Eighth International Conference on Tools with Artificial Intelligence, TAI'96, pages 183-193, Toulouse, France, 1996. IEEE Computer Society.

[11] D. Hernandez. Qualitative Representation of Spatial Knowledge, volume 804. Springer Verlag, 1994.

[12] A.G. Cohn, D.A. Randell, Z. Cui, and B. Bennet. Qualitative Spatial Reasoning and Representation. In P. Carrete and M.G. Singh, editors, Qualitative Reasoning and Decision Technologies, 1993.

[13] A. Galton. Continuous Change in Spatial Regions. In Spatial Information Theory: A Theoretical Basis for GIS (Proceedings of International Conference COSIT'97, pages 1-13. Springer-Verlag, 1994.

[14] T.K. Shih and A.Y. Chang. The Algebra of Spatio-temporal Intervals. In 1th Int. Conf. of Information Networking, pages 116-121. IEEE, 1998.

[15] P. Gagnon, Y. Bedard, and G. Edwards. Fundamentals of space and time and their integration inot forestry geographic databases. In Proceedings of IUFRO Conf. on the Integration of Forest Information Open Space and Time, pages 24-24, 1992.

[16] M. Vazirgiannis, Y. Theodoridis, and T. Sellis. Spatio-temporal composition and indexing for large multimedia applications. Multimedia Systems, 6(4):5-20, 1998.

[17] C. Claramunt and M. Theriault. Towards semantics for modelling spatio-temporal processing within gis. In Proceedings of the 719oth International Symposium on Spatial Data Handling, volume 2, pages 2.27-2.43, Charleston, 1996. IGU Commission of GIS.

[18] N. Tryfona and C.S. Jensesn. A Component-Based Conceptual Model for Spatiotemporal Design. Technical Report Choronchronos-CH-98-10, Chronochronos- A network of spatiotemporal database systems, 1998.

[19] El-Geresy B.A. and Abdelmoty A.I.. Order in Space: A General Formalism for Spatial Reasoning. Int. J. on Artificial Intelligence Tools, 6(4):423-450, 1997.

[20] D. Randell, Z. Cui, and A. Cohn. A Spatial Logic Based on Regions and Connection. In Principles of Knowledge Representation and Reasoning, KR'92, pages 165-176, 1992.

[21] Allen, J.F. and Hayes, P.J. Moments and points in an interval-based temporal logic. Technical Report TR 180, The University of Rochester, NY, 1987.

[22] M. Vilain and H. Kautz. Constraint Propagation Algorithms for Temporal Reasoning. In Proceedings of the Fifth National Conference on Artificial Intelligence AAAI-86, pages 377-382, 1986.

[23] D.A. Randell, A.G. Cohn, and Z. Cui. Computing Transitivity Tables: A Challenge for Automated Theorem Provers. In $C A D E$, Lecture Notes In Computer Science, 1992.

[24] J. Chomicki and P. Revesz. A Geometric Framework for Specifying Spatio-temporal Objects. In Proc. 6th International Workshop on Time Representation and Reasoning, 1999 . 


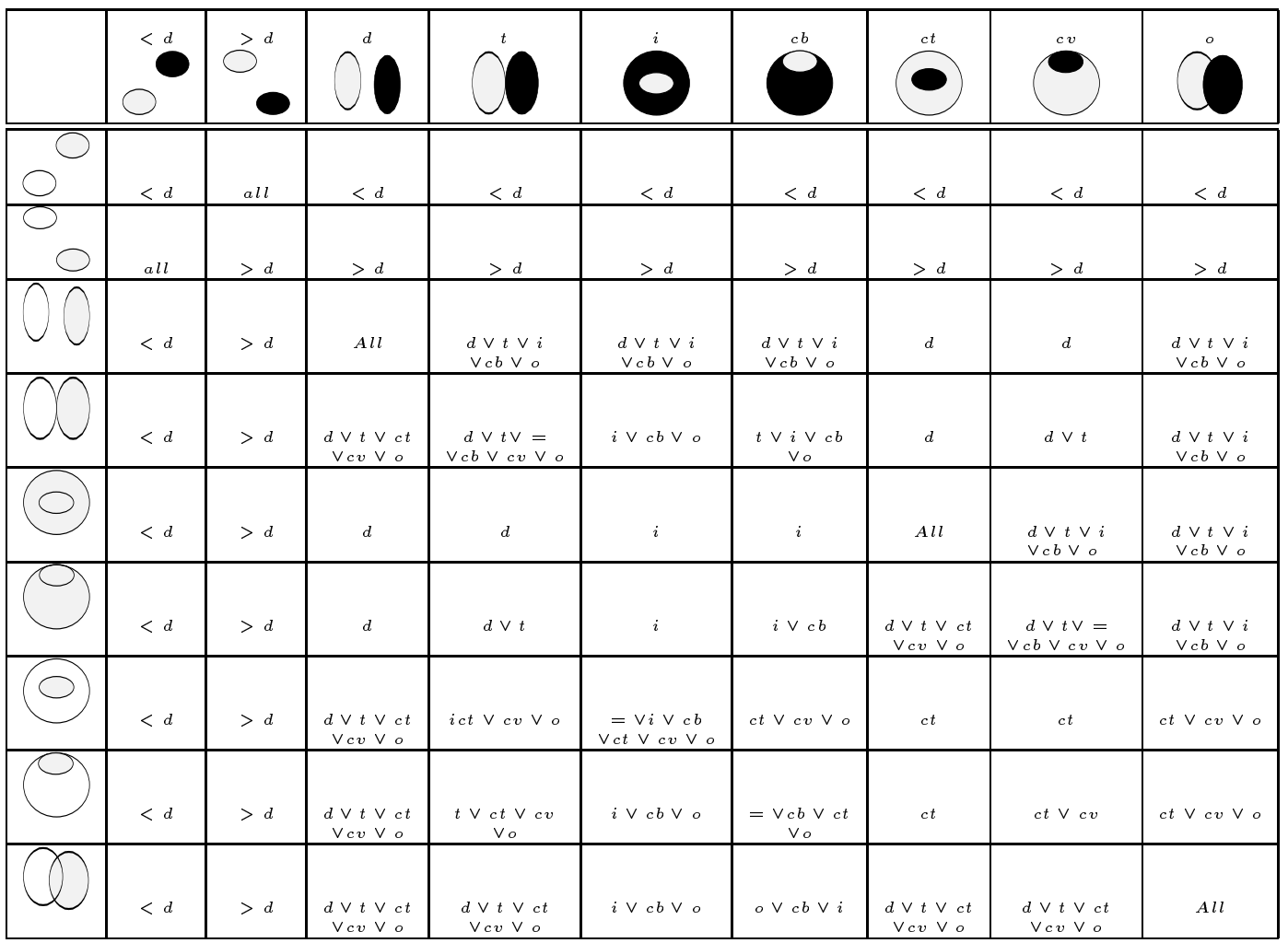

Table 1: The composition table between for spatio-temporal regions.

[25] T.Y. Jen and P. Boursier. A Model for Handling Topological Relationships in a 2D Environment. In Proceedings of the 6th international Symposium on Spatial Data Handling, volume 1, pages 73-89, 1994. 


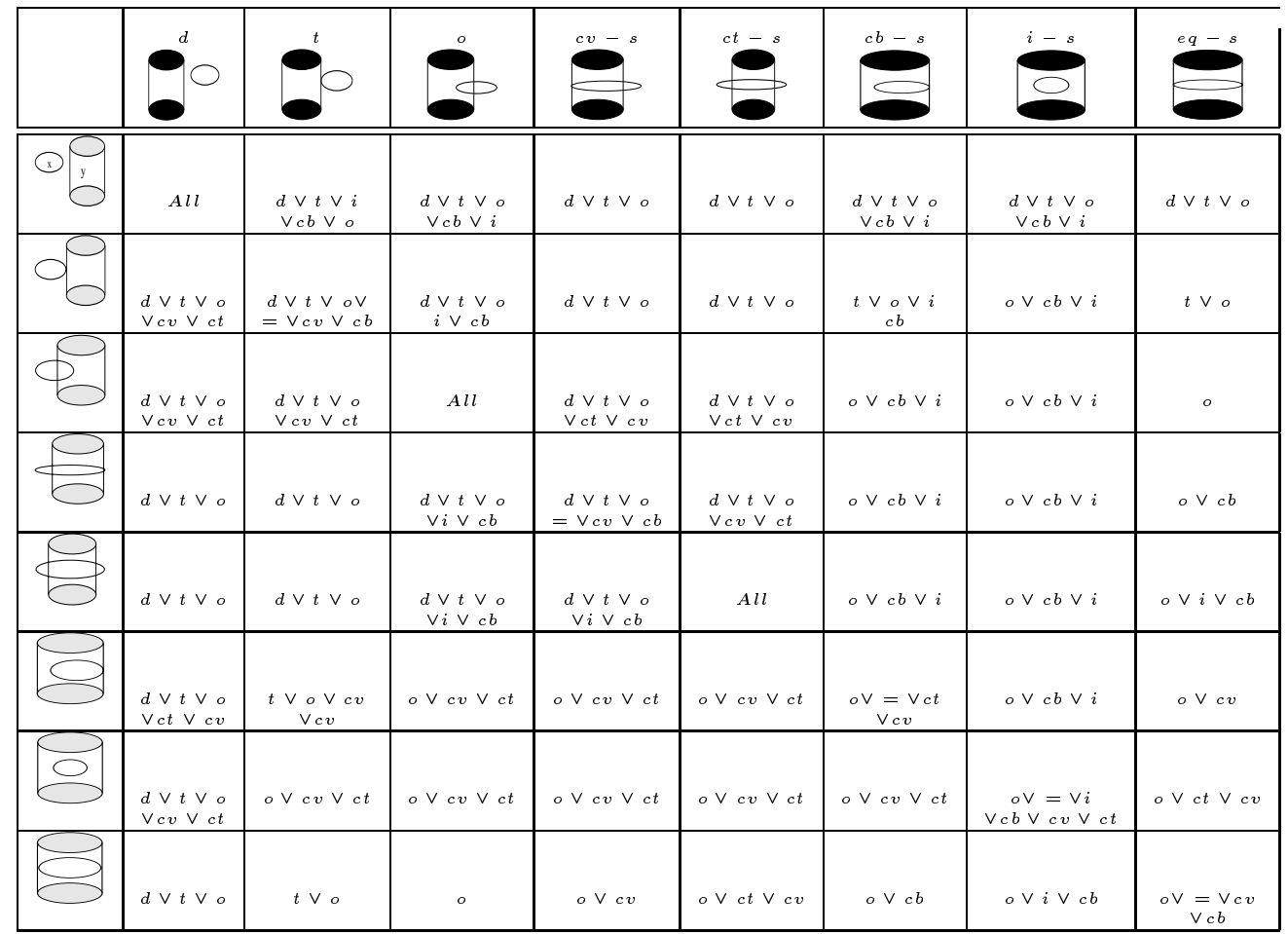

Table 2: The composition table between spatio-temporal regions (end-states of objects) and volumes (bodies of episodes). 